AIP Appilied Physics

\title{
Photoconductivity and characterization of nitrogen incorporated hydrogenated amorphous carbon thin films
}

Neeraj Dwivedi, Sushil Kumar, J. D. Carey, Hitendra K. Malik, and Govind

Citation: J. Appl. Phys. 112, 113706 (2012); doi: 10.1063/1.4768286

View online: http://dx.doi.org/10.1063/1.4768286

View Table of Contents: http://jap.aip.org/resource/1/JAPIAU/v112/i11

Published by the American Institute of Physics.

\section{Related Articles}

Determination of the deep donor-like interface state density distribution in metal/AI2O3/n-GaN structures from the photocapacitance-light intensity measurement

Appl. Phys. Lett. 101, 231608 (2012)

Controlled energy shuttling in terpolymers enabling independent optimization of absorption and transport properties in organic solar cell materials

Appl. Phys. Lett. 101, 231104 (2012)

Correlated evolution of barrier capacitance charging, generation, and drift currents and of carrier lifetime in $\mathrm{Si}$ structures during $25 \mathrm{MeV}$ neutrons irradiation

Appl. Phys. Lett. 101, 232104 (2012)

Deep level transient spectroscopy and minority carrier lifetime study on Ga-doped continuous Czochralski silicon Appl. Phys. Lett. 101, 222107 (2012)

Single crystal of LilnSe2 semiconductor for neutron detector

Appl. Phys. Lett. 101, 202101 (2012)

\section{Additional information on J. Appl. Phys.}

Journal Homepage: http://jap.aip.org/

Journal Information: http://jap.aip.org/about/about_the_journal

Top downloads: http://jap.aip.org/features/most_downloaded

Information for Authors: http://jap.aip.org/authors

\section{ADVERTISEMENT}

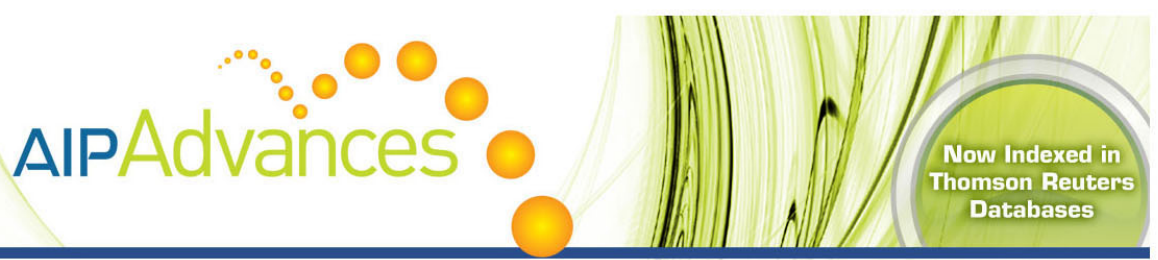

\section{Explore AIP's open access journal: Rapid publication \\ - Article-level metrics \\ Post-publication rating and commenting}




\title{
Photoconductivity and characterization of nitrogen incorporated hydrogenated amorphous carbon thin films
}

\author{
Neeraj Dwivedi, ${ }^{1,2}$ Sushil Kumar, ${ }^{1, a)}$ J. D. Carey, ${ }^{3}$ Hitendra K. Malik, ${ }^{2}$ and Govind ${ }^{1}$ \\ ${ }^{1}$ National Physical Laboratory (CSIR), K.S. Krishnan Road, New Delhi 110 012, India \\ ${ }^{2}$ Department of Physics, Indian Institute of Technology Delhi, New Delhi 110016, India \\ ${ }^{3}$ Advanced Technology Institute, University of Surrey, Guildford GU2 7XH, United Kingdom
}

(Received 22 August 2012; accepted 6 November 2012; published online 6 December 2012)

\begin{abstract}
The observation and origin of photoconductivity in high base pressure $\left(\sim 10^{-3}\right.$ Torr $)$ grown nitrogen incorporated hydrogenated amorphous carbon $(\mathrm{a}-\mathrm{C}: \mathrm{H}: \mathrm{N})$ thin films is reported. The magnitude of conductivity at room temperature was measured to increase by nearly two orders of magnitude and exhibits a maximum ratio of photoconductivity to dark conductivity of 1.5 as the nitrogen content increased to 15.1 at. \%. X-ray photoelectron spectroscopy, micro-Raman spectroscopy, and Fourier transform infrared spectroscopy reveal enhanced $\mathrm{sp}^{2}$ bonding at higher nitrogen contents. Residual film stress, Tauc band gap, hardness, and elastic modulus are all found to decrease with addition of nitrogen. The electrical characteristics suggest the creation of a-C:H:N/p-Si heterojunction diodes having rectifying behavior. The conductivity and electrical characteristics are discussed in term of band model, and the results show that high quality a-C:H:N films can be grown at high base pressures with properties comparable to those grown at low base pressures. (C) 2012 American Institute of Physics. [http://dx.doi.org/10.1063/1.4768286]
\end{abstract}

\section{INTRODUCTION}

Owing to their remarkable mechanical properties, hard hydrogenated amorphous carbon $(\mathrm{a}-\mathrm{C}: \mathrm{H})$ and unhydrogenated amorphous carbon (a-C), also called diamond-like carbon (DLC), thin films have attracted considerable attention. Along with the introduction of nitrogen, the resultant nitrogenated a-C:H (a-C:H:N) and a-C (a-C:N) thin films have shown the great promise with properties ${ }^{1}$ approaching that $\beta-\mathrm{C}_{3} \mathrm{~N}_{4}$, whose characteristics, such as hardness, have been found to be comparable to that of superhard diamond. Not only are the mechanical properties of a-C:H films of interest but also the electrical and optical properties have also been explored. Silva et al., ${ }^{2}$ Schwan et al., ${ }^{3}$ and Godet et al. ${ }^{4}$ have explored the electrical properties of a-C:H:N films and have suggested models which help to explain electron transport. Recently, we $\mathrm{e}^{5,6}$ have investigated some of the electrical properties of a-C:H:N and Ti/DLC multilayer films. For a-C:H:N films on a Si substrate we have shown the formation of a heterojunction along with improved electrical characteristics. ${ }^{5}$ The thin Ti layers present in the Ti/DLC multilayer structure help to promote nanostructured DLC film with sphere- and rod-like structures, which further give rise to enhance conductivity. ${ }^{6}$ If we look at the optoelectronic applications, c-Si/ $\mathrm{a}-\mathrm{C}: \mathrm{H}$ hetero- and a-C/a-C homojunction solar cells have been fabricated, ${ }^{7,8}$ though the understanding of the photoconduction mechanism remains to be resolved. Nonetheless, Konofaos et al. ${ }^{9}$ and Gao et al. ${ }^{10}$ have demonstrated the formation of diamond-like carbon/silicon heterojunction devices. Lazar et al. ${ }^{11}$ have developed a-C:H based metalinsulator-metal (MIM) and metal-insulator-semiconductor (MIS) structures and observed space charge limited conduc-

\footnotetext{
a) Author to whom correspondence should be addressed. Electronic mail: skumar@nplindia.org. Tel.: +91 11 45608650. Fax: +91 1145609310.
}

tion in their devices. While various other conduction mechanisms, which includes band tail hopping have also been reported $^{2-4}$ in films grown at low pressure, it remains important to understand the physics related to the conduction in a$\mathrm{C}: \mathrm{H}$ and modified a-C:H films especially those grown at high pressures.

A-C:H thin films are disordered semiconductors, in which the conduction and valence bands are not sharp. Such semiconductors show partially localized states near the Fermi level and in the band tails in addition to their extended states at higher energies. A-C:H films contain $\pi$ and $\pi^{*}$ states, associated predominantly with $\mathrm{sp}^{2}$ bonding, which lie inside the $\sigma$ and $\sigma^{*}$ states, associated with $\mathrm{sp}^{3}$ bonding. The electrical, optical, and optoelectronic properties of these films are governed by $\mathrm{sp}^{2}$ bonding, whereas $\mathrm{sp}^{3}$ bonding maintains the mechanical properties. ${ }^{12}$ The activation energy for conduction, estimated from electrical measurements, is an important parameter in determining the electron transport through disorder semiconductors. Silva et al. $^{2}$ have suggested that a shifting of $E_{F}$ with the nitrogen introduction can be a cause of changes in the activation energy. However, Ronning et al. ${ }^{13}$ have argued that nitrogen introduction increases the states, which changes the activation energy as well as conductivity. On the other hand, Godet et al. ${ }^{4,14}$ have suggested that nitrogen introduction causes a reduction of concentration of states, and this governs the conduction mechanism.

Although a-C:H and modified a-C:H films have been studied previously, the following issues need to be explored systematically in order to enhance their scientific and industrial impact:

(1) Photoconductivity (PC) in a-C:H films may be possible when there are electron-hole excitations between $\pi-\pi^{*}$ states. It is well known that a-C:H is generally a poor photoconducting material, since the photo-generated 
charge carriers have low mobility, are bound together, and tend to recombine leading to a high photoluminescence efficiency., ${ }^{3,15}$

(2) The a-C:H films exhibit high residual stress that leads to early delamination of the films from the substrate. For example, Schwan et al. $^{3}$ have observed very high stress, which varied from 6 to $12 \mathrm{GPa}$ in a-C:H films.

(3) The third issue is related to throughput and the cost. Usually a-C:H films are deposited at low base pressure $\left(10^{-5}\right.$ Torr or less), which requires sufficient pumping time and expensive vacuum equipment. For industrial applications the process should be simple, throughput should be high, and ultimately the product cost should be low. It is this final issue that lies at the core of this study where a higher base pressure is used in the deposition of the films.

Nitrogen has proved to be an important foreign element for a-C:H films that can help solve the first two problems mentioned above. Low residual stress in nitrogenated a-C:H (a-C:H:N) films, in which the residual stress is decreased from $4.6 \mathrm{GPa}$ to $1.5 \mathrm{GPa}$ with the increasing nitrogen partial pressure from $0 \%$ to $\sim 60 \%$, has been prepared previously. ${ }^{16}$ Silva $e t a l .^{2}$ have also observed decrease in residual stress from $\sim 5 \mathrm{GPa}$ to $1 \mathrm{GPa}$ with the increasing nitrogen content from 0 at. $\%$ to $\sim 15$ at. $\%$. Dwivedi et al. ${ }^{5}$ and Kumar et al. ${ }^{16}$ have realized the improved electrical and optical characteristics at low base pressure $\left(10^{-5}\right.$ Torr and less) deposited a-C:H:N films due to nitrogen incorporation. The cost effective a-C:H:N films can be deposited, without an use of turbo molecular pump, at a higher base pressure and offers advantages in terms of cost and speed of production. In the present study, higher base pressure $\left(\sim 10^{-3}\right.$ Torr $)$ deposited a-C:H:N films are studied for their electrical, optical, mechanical, and structural properties. The PC, dark conductivity (DC), activation energy, Tauc band gap, currentvoltage (I-V) characteristics, hardness, and residual stress are evaluated and correlated with the structure and the bonding environment within the films.

\section{EXPERIMENTAL DETAILS}

Nitrogen incorporated hydrogenated amorphous carbon (a-C:H:N) films were deposited on cleaned corning 7059 glass and p-type $\mathrm{Si}\langle 100\rangle$ wafers using asymmetric capacitive coupled radio frequency $13.56 \mathrm{MHz}$ plasma enhanced chemical vapor deposition (RF-PECVD). Besides standard three stage solvent cleaning, the substrates were also cleaned in an Ar plasma for $10 \mathrm{~min}$ prior to the deposition of films. Four sets of a-C:H:N films were deposited at different nitrogen partial pressure (NPP) of $0 \%(\mathrm{~S}-1), 44.4 \%$ (S-2), 66.6\% (S-3), and $76.1 \%$ (S-4), respectively, whereas all other process parameters, such as a negative self-bias of $100 \mathrm{~V}$, were kept constant.

The thickness of the films was measured using a TaylorHobson Talystep instrument and found to be $276 \mathrm{~nm}$, $244 \mathrm{~nm}, 195 \mathrm{~nm}$, and $162 \mathrm{~nm}$ in samples S-1, S-2, S-3, and S4, respectively. Keithley solid state electrometer (610C) was used to measure both the DC and PC. The PC was evaluated by exposing the samples to a photon power density of
$100 \mathrm{~mW} / \mathrm{cm}^{2}$, where the samples were metalized into coplanar structures. The surface composition and the bonding of the films was investigated by X-ray photoelectron spectroscopy (XPS) (Perkin Elmer, USA, model no. 1257), and timeof-flight secondary ion mass spectroscopy (ToF-SIMS) (ION-TOF GmBH, Germany) was used to analyze the bulk composition and depth profile of the films. Micro-Raman measurements were carried out on these films using a Renishaw inVia reflex micro-Raman spectrometer employing an excitation wavelength of $514.5 \mathrm{~nm}$. Fourier transform infrared (FTIR) spectroscopy (model Perkin Elmer spectrum $\mathrm{BX}$ ) analysis was performed to investigate the bonding environment. The optical measurements were performed using Schimadzu ultra-violet (UV-Vis) spectrometer for determination of the Tauc band gap $\left(\mathrm{E}_{\mathrm{g}}\right)$. In order to evaluate $\mathrm{E}_{\mathrm{g}}$, first the absorption coefficient $(\alpha)$ was estimated using the relation $\alpha=1 / \mathrm{d}[\ln \{(1-\mathrm{R}) / \mathrm{T}\}]$, where $\mathrm{d}, \mathrm{R}$, and $\mathrm{T}$ are the thickness, reflection, and transmission, respectively. A (Tauc) plot $(\alpha \mathrm{h} \nu)^{1 / 2}$ versus $\mathrm{h} \nu$ gives the values of $\mathrm{E}_{\mathrm{g}}$. The residual stress (S) in these films was measured using 500TC temperature controlled film stress measurement system (FSM Frontier Semiconductor, USA). We calculated the residual stress using Stoney formula, as

$$
\mathrm{S}=\frac{E_{s} d_{s}^{2}}{6\left(1-\nu_{s}\right) d_{f}}\left(\frac{1}{R_{f}}-\frac{1}{R_{0}}\right)
$$

where $\mathrm{E}_{\mathrm{s}}, \nu_{\mathrm{s}}, \mathrm{d}_{\mathrm{f}}$, and $\mathrm{d}_{\mathrm{s}}$ are Young's modulus, Poisson ratio, thickness of film, and thickness of substrate, respectively, and $R_{0}$ and $R_{f}$ are the radii of substrate curvature before and after film deposition. Fully automatic software controlled IBIS nanoindentation (Fisher-Cripps laboratories Pvt. Limited, Australia) with a diamond Berkovich indenter at indentation load of $10 \mathrm{mN}$ was employed to measure the nanomechanical properties of the a-C:H:N films.

\section{RESULTS AND DISCUSSION}

\section{A. XPS analysis}

XPS is an important technique for investigation of surface composition and chemical analysis. The XPS spectra in the binding energy range of $200-600 \mathrm{eV}$ for a-C:H:N films deposited at NPPs of $0 \%$ (S-1) and $76.1 \%$ (S-4) reveal the presence of $\mathrm{C}$ and $\mathrm{O}$ atoms. The presence of nitrogen atoms is only observed in sample S-4. Figures 1(a)-1(c) show the XPS core level spectra of C1s and N1s for different a-C:H:N films. The nitrogen content of sample S-4 was estimated using the ratio of area of peaks obtained from the N1s and $\mathrm{C} 1 \mathrm{~s}$ core level spectra, i.e., $\mathrm{N} /(\mathrm{N}+\mathrm{C})=\left(\mathrm{A}_{\mathrm{N}} / 0.38\right) /\left(\mathrm{A}_{\mathrm{N}} / 0.38+\mathrm{A}_{\mathrm{C}} / 0.205\right)$, where $A_{N}$ and $A_{C}$ are the areas under the N1s and $C 1$ s core level spectra and the constants 0.38 and 0.205 are the atomic sensitivity factors of nitrogen and carbon, respectively. ${ }^{17}$ The amount of nitrogen in sample S-4 was found to be 15.1 at. \%. The core level spectra were deconvolved to examine the bonding states of $\mathrm{C}$ with $\mathrm{C}, \mathrm{N}$, and $\mathrm{O}$; Figure 1(a) shows the deconvolved $\mathrm{C} 1 \mathrm{~s}$ core level spectra of sample $\mathrm{S}-1$. The peaks centered at $284.1 \mathrm{eV}$ and $285.1 \mathrm{eV}$ represent the $\mathrm{sp}^{2}$ and $\mathrm{sp}^{3}$ hybridized carbon-carbon bonding. The peak located at $286.3 \mathrm{eV}$ shows the bonding state of carbon with oxygen. 

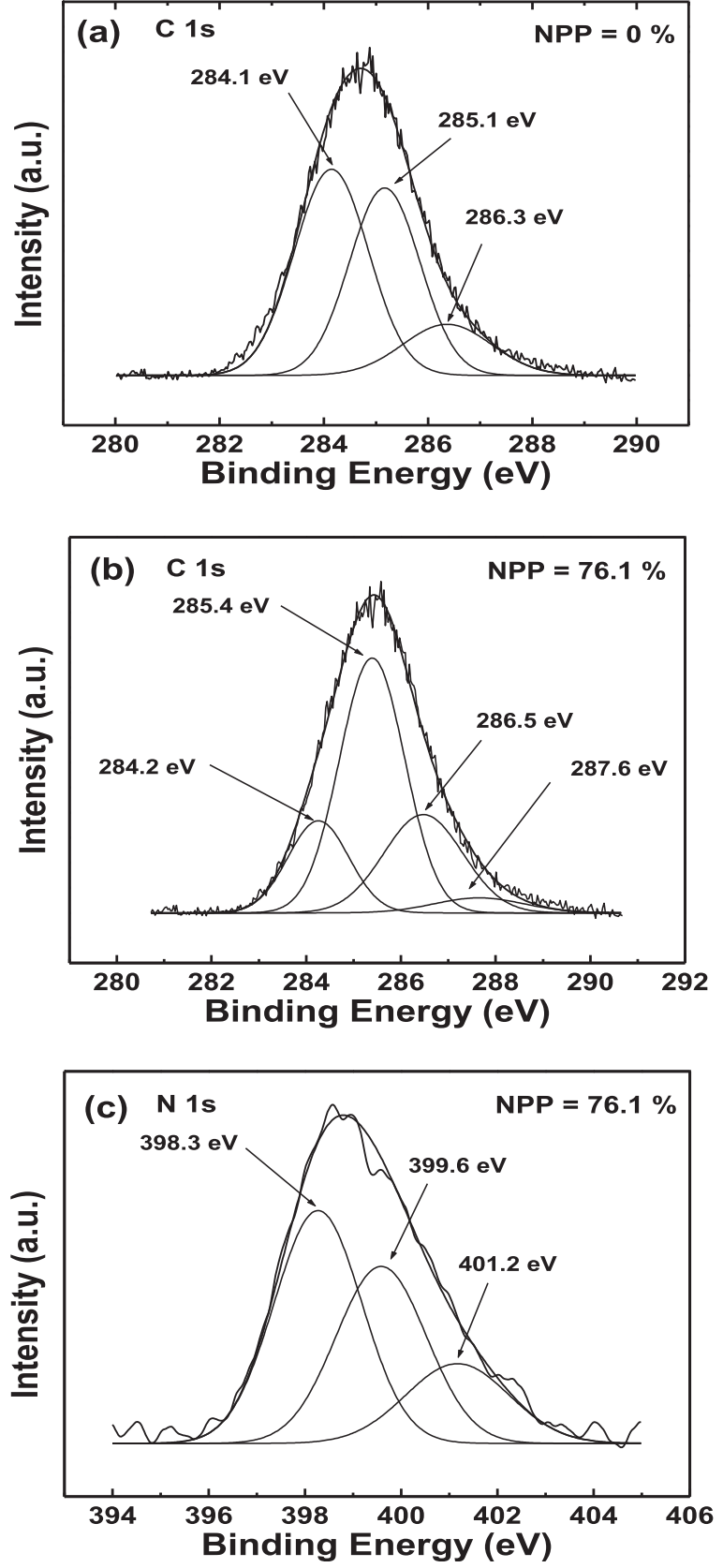

FIG. 1. XPS core level spectra of (a) C 1s for sample S-1, (b) C 1s for sample S-4 and (c) N 1s for sample S-4.

During the peak fitting, the full width at half maximum (FWHM) values of these peaks was kept at 1.67, 1.6, and $2.0 \mathrm{eV}$, respectively. Similarly, the deconvolved C1s core level spectrum of sample S-4 was fitted with four peaks as shown in Fig. 1(b). A low intense peak centered at $284.2 \mathrm{eV}$ is generally labeled as adventitious carbon due to atmospheric contamination and peaks located at the positions of $285.4 \mathrm{eV}$ and $286.5 \mathrm{eV}$ represent the $\mathrm{sp}^{2}$ and $\mathrm{sp}^{3}$ hybridized carbon-nitrogen bondings. The low intensity peak centered at $287.6 \mathrm{eV}$ shows evidence of $\mathrm{N}-\mathrm{O}$ or $\mathrm{C}-\mathrm{O}$ bondings. The deconvolved $\mathrm{N} 1 \mathrm{~s}$ core level spectrum of sample S-4 is shown in Fig. 1(c). This spectrum was fitted with three peaks located at $398.3 \mathrm{eV}$ ( $\mathrm{C}=\mathrm{N}-\mathrm{C}$ bonding), $399.6 \mathrm{eV}$ ( $\mathrm{sp}^{3}$ carbon-nitrogen bonding), and $401.2 \mathrm{eV}\left(\mathrm{sp}^{2}\right.$ carbon-nitrogen bonding), respectively. For sample S-4, the peaks centered at $284.2 \mathrm{eV}$ and $285.4 \mathrm{eV}$ in
C1s spectra are found to be analogous to that of the peak centered at $401.2 \mathrm{eV}$ and $399.6 \mathrm{eV}$ in N1s spectra, which show the carbon-nitrogen bonding in $\mathrm{sp}^{2}$ and $\mathrm{sp}^{3}$ hybridization, respectively. By XPS analysis it is confirmed that introduction of nitrogen in a-C:H:N films enhances the graphite-like $\mathrm{sp}^{2}$ bonding.

\section{B. ToF-SIMS and deposition rate analyses}

Since XPS is surface sensitive technique, ToF-SIMS measurement was performed to analyze the bulk composition. SIMS also provides information about film/substrate interface and depth profile of the elements. Typical ToFSIMS depth profiles for $\mathrm{C}, \mathrm{H}, \mathrm{N}, \mathrm{O}$, and $\mathrm{Si}$ from a-C:H:N film deposited at NPP of $76.1 \%$ (sample S-4) are shown in Fig. 2. It is evident from the figure that $\mathrm{C}, \mathrm{H}$, and $\mathrm{N}$ atoms are present with the incorporation of $\mathrm{O}$ as an impurity in the film. It is generally realized that during the growth of pure a$\mathrm{C}$ and a-C:H films, the $\mathrm{C}$ ions strike the substrate with high energy and some of them diffuse into the substrate. This may lead to the formation of mixed a-SiC and a-SiC:H kind of layers at the interface. However, compared with a-C:H films, ${ }^{6}$ the ToF-SIMS depth profile of a-C:H:N film deposited at a NPP of $76.1 \%$ reveals negligible interdiffusion of C into the Si substrate. Thus, it is realized that a high NPP restricts the interdiffusion of $\mathrm{C}$ into $\mathrm{Si}$. By contrast, $\mathrm{H}$ is significantly diffused into the substrate $\mathrm{Si}$ due to its low atomic weight. A small amount of $\mathrm{O}$ was found in the a-C:H:N film due to atmospheric contamination. It is to be noted that $\mathrm{O}$ is present during the depth profiling of substrate $\mathrm{Si}$, which may be due to the fact that Czochralski technique based $\mathrm{Si}$ wafers possess $\mathrm{O}$ as an impurity.

The deposition rate of a-C:H:N films was also determined and found to vary inversely with NPP. When NPP (nitrogen content) was increased from $0 \%$ to $76.1 \%$ (0 at. $\%-15.1$ at. \%) deposition rate decreased from $42 \mathrm{~nm} / \mathrm{min}$ to $25 \mathrm{~nm} / \mathrm{min}$. During growth of a-C:H:N films, there is a competition between growth and etching due to ionic bombardment. An increase in the NPP enhances the nitrogen species in carbon-nitrogen plasma, which further increases the ionic bombardment. This results in more etching of the growing surface and, hence, a lower deposition rate.

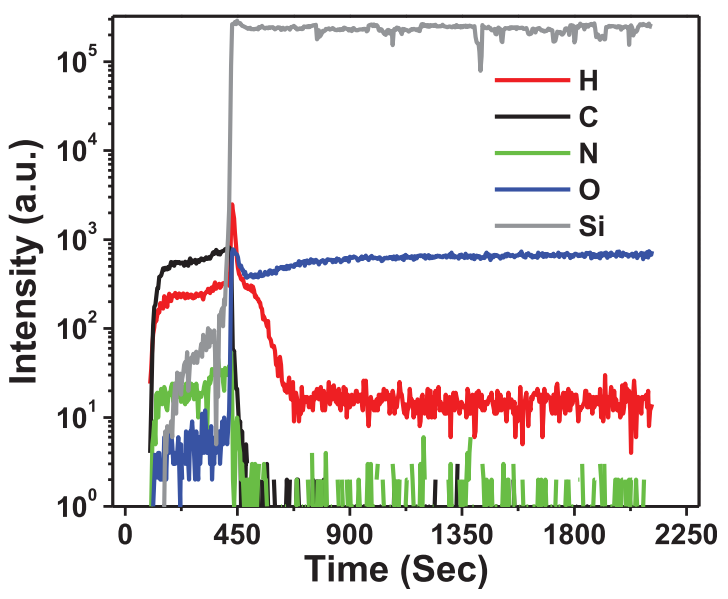

FIG. 2. ToF-SIMS depth profile of a-C:H:N film grown at NPP of $76.1 \%$. 


\section{Micro-Raman spectroscopic and FTIR analyses}

Raman spectroscopy is a powerful non-destructive tool for investigating the bonding in carbon based materials. The excitation wavelength of $514.5 \mathrm{~nm}$ (energy $\sim 2.4 \mathrm{eV}$ ) is used here for micro-Raman measurements, which can only probe directly the $\mathrm{sp}^{2}$ phase of a-C:H:N films. The Raman spectra of a-C:H and a-C:H:N films exhibit two main features; the D peak $\left(\sim 1350 \mathrm{~cm}^{-1}\right)$ and $G$ peak $\left(\sim 1550 \mathrm{~cm}^{-1}\right)$. Here $D$ stands for a disorder attributed peak due to breathing modes of six fold $\mathrm{sp}^{2}$ aromatic clusters. The $\mathrm{G}$ peaks arises from vibrations between in $\mathrm{sp}^{2}$ carbon atoms. Ferrari and Robertson ${ }^{18}$ have discussed the origin of the D peak and have suggested that the visible Raman spectra of amorphous carbon films depend on bond length and bond angle disorder, $\mathrm{sp}^{2}$ rings or chains and the $\mathrm{sp}^{3} / \mathrm{sp}^{2}$ ratio. Hence, because of variations of the $\mathrm{G}$ peak position and the $\mathrm{I}_{\mathrm{D}} / \mathrm{I}_{\mathrm{G}}$ intensity ratio, change in the $\mathrm{sp}^{2}$ phase can be probed. Figures 3(a) and 3(b) show the Raman spectra of samples S-1 and S-4. The spectra clearly reveal the shifting of $\mathrm{G}$ band towards higher wavenumber with an increase in NPP from $0 \%$ to $76.1 \%$, which is due to an enhancement in the $\pi-\pi^{*} \mathrm{sp}^{2}$ bonding. The Raman spectra of these films are fitted with two Gaussian functions in order to investigate how the $\mathrm{I}_{\mathrm{D}} / \mathrm{I}_{\mathrm{G}}$ ratio and the $\mathrm{G}$ peak position change by varying the NPP. For sample S-1, the G peak is found at $1536 \mathrm{~cm}^{-1}$ which shifts to $1548 \mathrm{~cm}^{-1}$ in sample S-4 upon nitrogen incorporation. This Raman shift of $\sim 12 \mathrm{~cm}^{-1}$ towards higher wavenumber indicates nitrogen
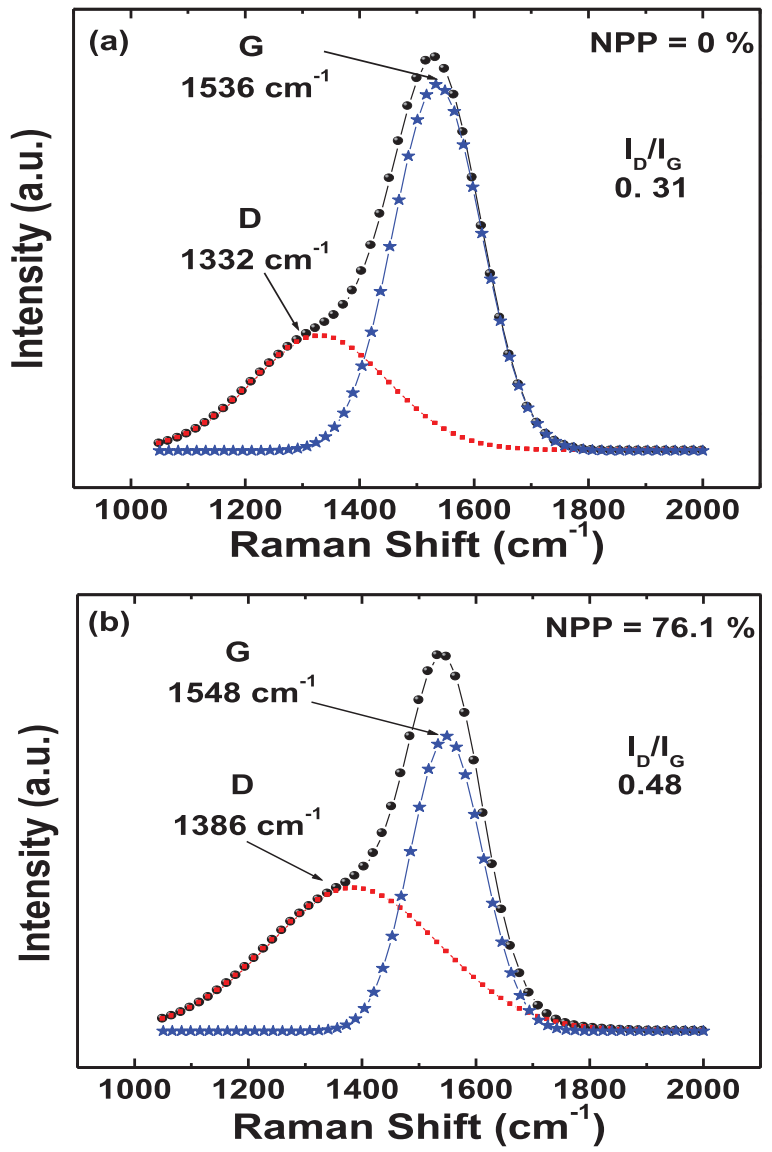

FIG. 3. Deconvolved visible $(5124 \mathrm{~nm})$ Raman spectra of sample (a) S-1 and (b) S-4 driven enhancement of $\mathrm{sp}^{2}$ bonding. In addition nitrogen incorporation in a-C:H:N film makes the intensity of the D peak higher due to an enhancement in disorder resulting from substitution of $\mathrm{N}$ atoms in aromatic $\mathrm{sp}^{2}$ clusters. This explains why the $\mathrm{I}_{\mathrm{D}} / \mathrm{I}_{\mathrm{G}}$ ratio increases from 0.31 to 0.48 with the increasing NPP from $0 \%$ to $76.1 \%$.

The FTIR spectra of samples S-1 and S-4 are shown in Figs. 4(a) and 4(b), respectively. A strong $\mathrm{CO}_{2}$ peak due to atmospheric contamination was observed in these films; since this peak has no physical significance in relation to the properties of interest, the region $2230-2410 \mathrm{~cm}^{-1}$ has been removed from the FTIR spectra. The FTIR spectra from sample $\mathrm{S}-1$ shows the presence of $\mathrm{sp}^{\mathrm{n}} \mathrm{C}-\mathrm{H}_{\mathrm{x}}$ (where $\mathrm{n}=2,3$ and $\mathrm{x}=1$, 2, and 3) stretching modes in the range 2800$3100 \mathrm{~cm}^{-1}$. The band in the range $1560-1660 \mathrm{~cm}^{-1}$ reveals the presence of $\mathrm{C}=\mathrm{C}$ bonding only. When the NPP (nitrogen content) was increased to $76.1 \%(15.1$ at. \%) the band in the range $1560-1660 \mathrm{~cm}^{-1}$ becomes stronger with a sharp peak at $\sim 1600 \mathrm{~cm}^{-1}$. This is attributed to increased $\mathrm{sp}^{2}$ phase due to $\mathrm{C}=\mathrm{N}$ bonding (amino group).

\section{Residual stress, hardness, and elastic modulus analyses}

The presence of residual stress $(\mathrm{S})$ is a major issue in a$\mathrm{C}: \mathrm{H}$ films and excess stress leads to delamination of the films from the substrate. High values of stress restricts the growth of comparatively thick a-C:H films and limits its use in industrial applications. Nitrogen incorporation in a-C:H films has,
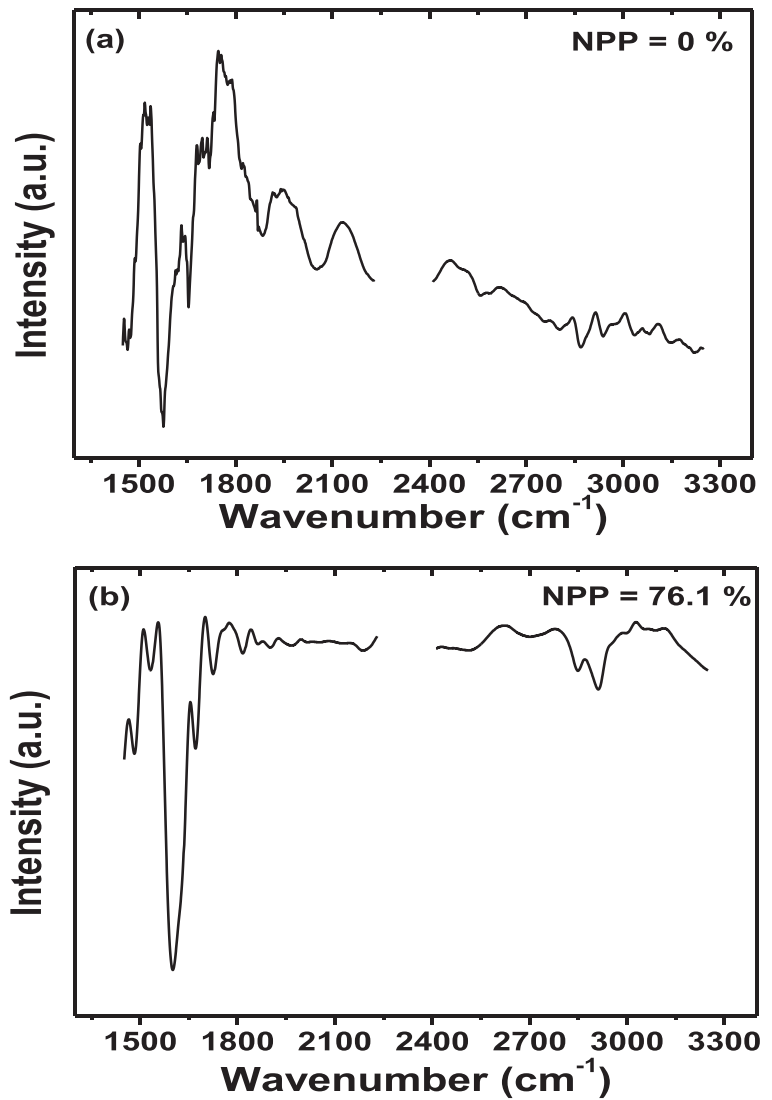

FIG. 4. FTIR spectra of a-C:H:N films grown at NPP of (a) $0 \%$ and (b) $76.1 \%$. 
however, evolved as an important way to reduce excessive stress. The values of $\mathrm{S}$ in a-C:H:N films were measured to be in the range between $0.8 \mathrm{GPa}$ and $1.9 \mathrm{GPa}$ (Fig. 5(a)) and scale inversely with nitrogen partial pressure. In addition, Figures 5(b) and 5(c) show the variation of hardness $(H)$ and elastic modulus (E), respectively, with NPP for different a$\mathrm{C}: \mathrm{H}: \mathrm{N}$ films. The values of $H$ and $\mathrm{E}$ in these films were found to be significantly high and varied in the range $38-22 \mathrm{GPa}$ and 462-330 GPa, respectively, with the increasing nitrogen content. The observed reduction in $\mathrm{S}$ and $H$ with an enhancement in NPP was due to the increased $\mathrm{sp}^{2}$ bonding. Such a$\mathrm{C}: \mathrm{H}: \mathrm{N}$ films with high value of $H$ may be useful for their hard, protective, encapsulate and anti-reflection coating on silicon solar cells.

\section{E. Tauc band gap analysis}

Figure 6 shows the variation of the Tauc band gap $\left(E_{g}\right)$ with nitrogen partial pressure, where the value of $\mathrm{E}_{\mathrm{g}}$ was found to decrease from $2.2 \mathrm{eV}$ to $1.45 \mathrm{eV}$ with the increasing NPP (nitrogen content) from $0 \%$ to $76.1 \%$ ( 0 at. \%-15.1 at. \%). The
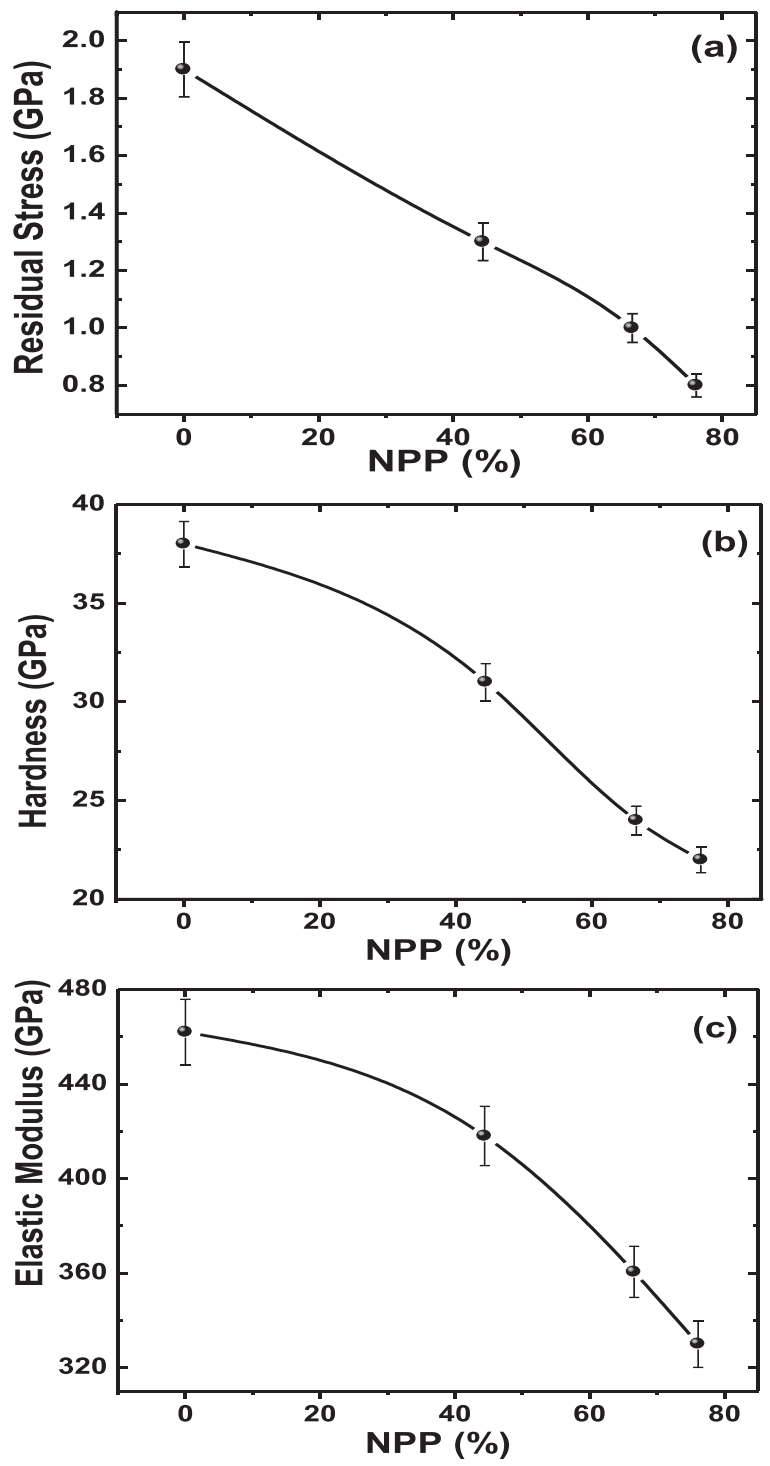

FIG. 5. Variation of (a) residual stress, (b) hardness, and (c) elastic modulus with NPP for a-C:H:N films.

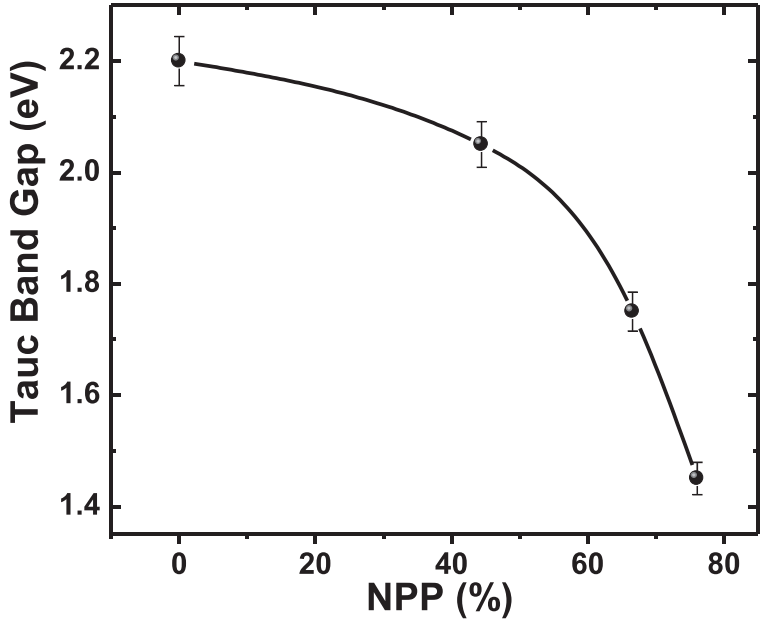

FIG. 6. Variation of Tauc band gap with NPP for a-C:H:N films.

value of $\mathrm{E}_{\mathrm{g}}$ in a-C:H:N films strongly depend on $\mathrm{sp}^{2}$ clusters size and the bonding. The nitrogen incorporation in a-C:H:N films takes place at the expense of carbon from $\mathrm{C}-\mathrm{C}$ bonding, which promotes the formation of $\mathrm{sp}^{2}$ bonding ${ }^{19}$ and reduces $\mathrm{E}_{\mathrm{g}}$. Within the cluster model ${ }^{20}$ the value of $\mathrm{E}_{\mathrm{g}}$ in amorphous carbon depends on six fold rings in the clusters and can be estimated from $\mathrm{E}_{\mathrm{g}}=2 \gamma / \mathrm{M}^{1 / 2}$, where $\gamma$ is the nearest neighbour $\pi$ interaction and $\mathrm{M}$ is the number of six-fold rings in the clusters. Thus, an increase in the size of six-fold $\pi$ bonded $\mathrm{sp}^{2}$ clusters embedded in amorphous $\mathrm{sp}^{3}$ matrix reduces $\mathrm{E}_{\mathrm{g}}$ of amorphous carbon films.

\section{F. Temperature dependent conductivity analysis}

The electrical transport of a-C:H:N films strongly depends on the $\mathrm{sp}^{2}$ bonding, where $\pi-\pi^{*}$ states lie inside $\sigma-\sigma^{*}$ states and control the conduction of charge carriers. Figure 7(a) shows the variation of conductivity (DC and PC) with inverse temperature for various a-C:H:N films. Here the conductivity was found to increase with increasing temperature consistent with semiconducting behaviour. At zero nitrogen incorporation (sample S-1), the DC varied from $\sim 4.6 \times 10^{-11}$ to $2.7 \times 10^{-9} \Omega^{-1} \mathrm{~cm}^{-1}$ when the temperature was raised from $303 \mathrm{~K}$ to $473 \mathrm{~K}$. However, no PC was observed in this film as light-generated electron-hole pairs readily recombine due to the low mobility of charge carriers. The magnitude of conductivity (both DC and PC) was enhanced as the nitrogen content increased. At low or zero nitrogen content, the concentration of $\mathrm{sp}^{2}$ clusters is small and they are distributed in a random manner with a large separation between neighbouring clusters in an a-C:H matrix. The large separation between clusters reduces transport by hopping, resulting in low DC and no PC and explains why sample S-1 with a large band gap of $2.2 \mathrm{eV}$ exhibits low DC and no PC. However, at higher nitrogen content, the concentration and size of $\mathrm{sp}^{2}$ clusters in amorphous a-C:H matrix increases and the separation between the clusters reduces; ${ }^{24}$ this lowers the band gap, increasing the DC and PC. This explains why sample S-4 with 15.1 at. \% nitrogen content with a lower band gap of $1.45 \mathrm{eV}$ shows high DC and maximum PC. 

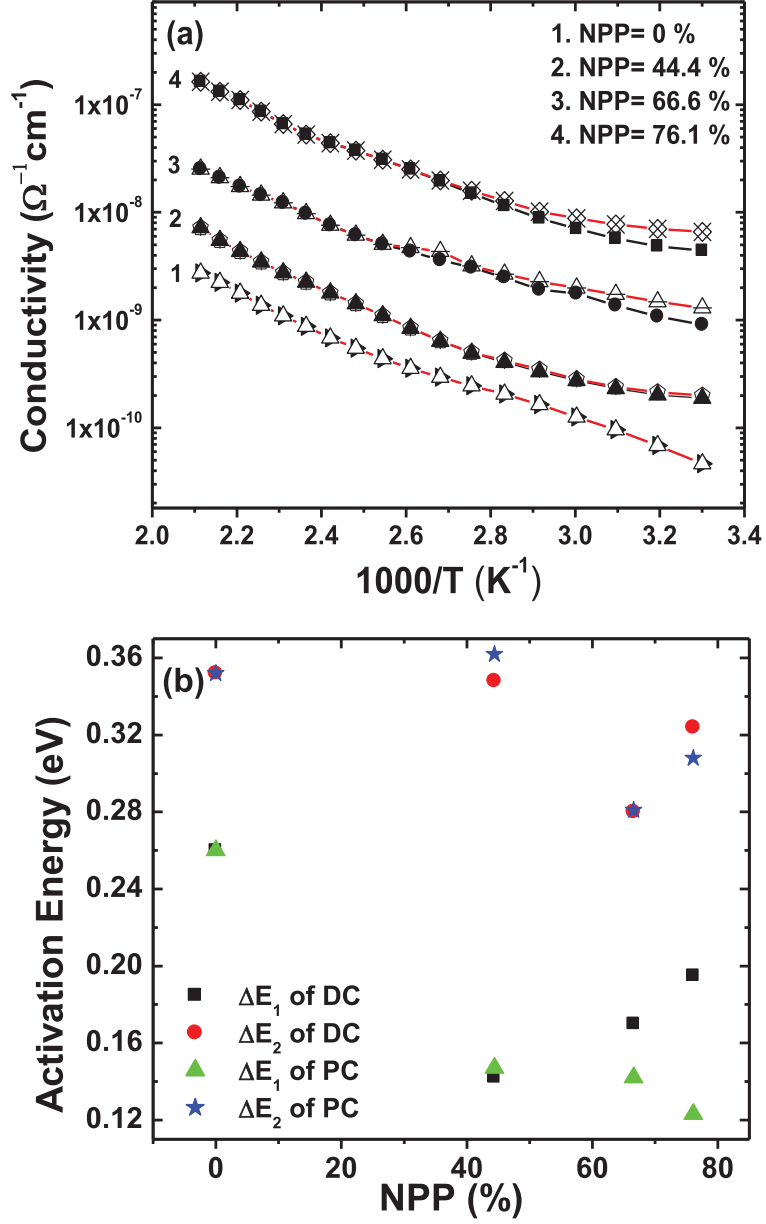

FIG. 7. (a) Variation of conductivity (DC and PC) with inverse of temperature for a-C:H:N films. The filled symbols with black line represent DC and open symbols with red line represent PC. (b) Variation of activation energy (for DC and PC) for a-C:H:N films.

In these films PC was only observed at lower temperatures because at higher temperatures the magnitude of DC itself swamped the PC signal. Recently, Umeno and Adhi$\mathrm{kari}^{21}$ have examined the conductivity of a-C:H:N films under dark and light conditions but photoconductivity only observed in their study at low temperatures with a PC/DC ratio $(\sim 1.2)$ at room temperature. Liu et al. ${ }^{22}$ have explored the electrical properties of nitrogen incorporated ta-C (ta$\mathrm{C}: \mathrm{N})$ films and found only a small photoconductivity with maximum $\mathrm{PC} / \mathrm{DC}$ ratio $\sim 1.03$. Somani et al. ${ }^{23}$ have studied the electrical conductivity of a-C:H:N films under dark and light (AM 1.5) conditions. They have found very weak photovoltaic effect even when they reduced the thickness of a$\mathrm{C}: \mathrm{H}: \mathrm{N}$ films. We obtained maximum $\mathrm{PC} / \mathrm{DC}$ ratio $\sim 1.5$ at room temperature in sample S-4. Hence, in view of the observed photoconductivity in our films grown at high pressure seems to be very encouraging and these a-C:H:N films could be employed in photovoltaic applications.

The activation energy ( $\Delta \mathrm{E})$ for conduction is a very important electrical parameter to understand the transport mechanism. An Arrhenius plot of conductivity with temperature for each of these a-C:H:N films reveals two slopes: one in the lower temperature range and another in the higher temperature range, which infers the two modes of conduction with two activation energies $\Delta \mathrm{E}_{1}$ and $\Delta \mathrm{E}_{2}$. Therefore, the resultant conductivity is given by

$$
\sigma=\sigma_{01} \exp \left(-\Delta \mathrm{E}_{1} / \mathrm{kT}\right)+\sigma_{02} \exp \left(-\Delta \mathrm{E}_{2} / \mathrm{kT}\right)
$$

where $\sigma_{01}$ and $\sigma_{02}$ are conductivity prefactors, $\mathrm{k}$ is Boltzmann's constant, and $\mathrm{T}$ is the temperature in Kelvin. Figure 7(b) shows the variation of activation energy ( $\Delta \mathrm{E}_{1}$ and $\left.\Delta \mathrm{E}_{2}\right)$ of DC and PC with nitrogen partial pressures. Since the slope was found to be smaller (larger) in the lower (higher) temperature range, the first (second) term of Eq. (2) is dominant and the conductivity is governed by the activation energy $\Delta \mathrm{E}_{1}\left(\Delta \mathrm{E}_{2}\right)$. The behaviour of the activation energy is discussed in terms of band structure (Fig. 8). We interpret $\Delta \mathrm{E}$ as the energy difference between the $\mathrm{E}_{\mathrm{F}}$ and the conducting states. In disordered semiconductors, several localized states exist near the Fermi level as well as in the band tails. These semiconductors also contain extended states and the activation energy directly reflects the type of conduction. In the low temperature regime where the activation energy is found to be comparatively lower $\left(\Delta \mathrm{E}_{1}\right)$ in the range $0.14-0.26 \mathrm{eV}$, the conduction takes place in localized states, situated at or very close to the Fermi level. However, at higher temperature regime where activation energy is found to be higher $\left(\Delta \mathrm{E}_{2}\right)$ in the range $0.28-0.35 \mathrm{eV}$, the conduction occur either through states situated in the band tails or through extended states. Silva et $a .^{2}$ have also obtained same behaviour of activation energy in low and high temperature regimes. They have found $\Delta \mathrm{E}$ between $0.34 \mathrm{eV}$ and $0.55 \mathrm{eV}$ in low temperature regime. In our case, the smaller activation energy in the lower and higher temperature regimes may be attributed to presence of large number of $\mathrm{sp}^{2}$ clusters separated by a narrower gap due to which electrons easily hope between the two successive clusters due to nitrogen incorporation. In addition, following important points should be kept in mind:

(i) Nitrogen may act as an n-type dopant for a-C:H films and its incorporation shifts the Fermi level $\left(\mathrm{E}_{\mathrm{F}}\right)$ towards the conduction band.

(ii) As-deposited amorphous carbon films show weakly ptype behaviour as $\mathrm{E}_{\mathrm{F}}$ in these films lie slightly below the mid gap. ${ }^{3}$

(iii) Since disorder semiconductor (such as a-C:H or a$\mathrm{C}: \mathrm{H}: \mathrm{N})$ contains several states near the Fermi level as well as in band tails as suggested elsewhere, ${ }^{20,24}$ the introduction of nitrogen in a-C:H:N films may be altered (increase or decrease) the size and concentration of these $\mathrm{sp}^{2}$ states.

The activation energy may also be modified either due to the shifting of the Fermi level or due to the modification of the states. We schematically propose a model, shown in Fig. 8, where Fermi level is initially situated slightly below the mid-gap level for a-C:H:N film at zero nitrogen content but gets shifted towards conduction band with an increase of nitrogen content. The value of $\Delta \mathrm{E}_{1}$ and $\Delta \mathrm{E}_{2}$ (DC) for a$\mathrm{C}: \mathrm{H}: \mathrm{N}$ film grown at NPP of $0 \%$ (sample $\mathrm{S}-1$ or pure a-C:H film) was found to be $0.26 \mathrm{eV}$ and $0.352 \mathrm{eV}$, respectively. This decreased to $0.142 \mathrm{eV}$ and $0.348 \mathrm{eV}$, respectively, for aC:H:N film deposited at NPP of $44.4 \%$ (sample S-2). With 


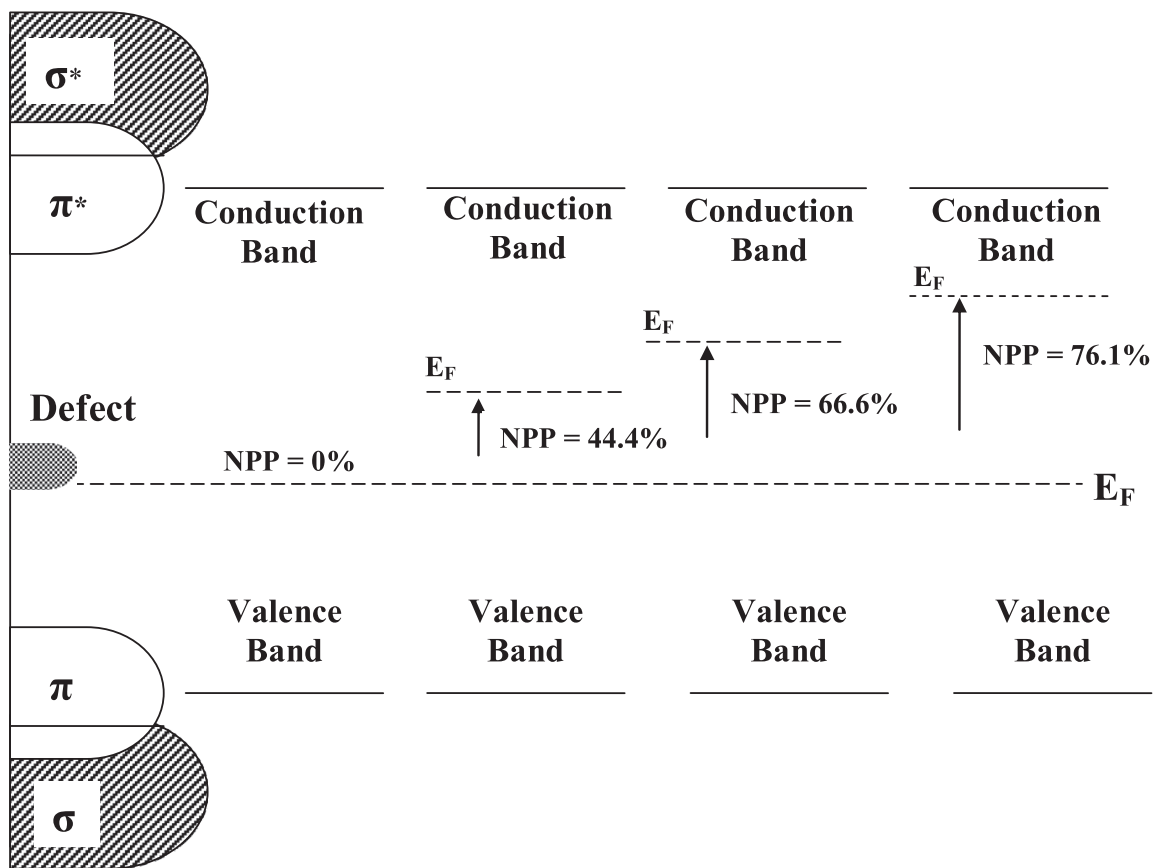

FIG. 8. Proposed model, showing shifting of Fermi level towards conduction band with the increase of NPP. This model was sketched on the basis of observed variation in electrical and optical properties with the changing NPP of a-C:H:N films. the increase of NPP from $0 \%$ to $44.4 \%, \mathrm{E}_{\mathrm{F}}$ shifts towards the conduction band and the energy difference between $\mathrm{E}_{\mathrm{F}}$ and the conducting states is reduced, which results in reduced activation energies. The activation energies $\Delta \mathrm{E}_{1}$ and $\Delta \mathrm{E}_{2}$ (DC) for a-C:H:N film grown at NPP of $66.6 \%$ (sample $\mathrm{S}-3$ ) are found to be $0.17 \mathrm{eV}$ and $0.28 \mathrm{eV}$, respectively. In sample S-3, $\Delta \mathrm{E}_{1}$ is found to be lower than in sample $\mathrm{S}-1$ but higher than $\Delta \mathrm{E}_{1}$ of sample $\mathrm{S}-2$. On the other hand, $\Delta \mathrm{E}_{2}$ is found to be lower than that of $\Delta E_{2}$ of $S-1$ and $S-2$. It was expected that at this NPP, $E_{F}$ has shifted such that the carriers need only $\sim 0.17 \mathrm{eV}$ from $\mathrm{E}_{\mathrm{F}}$ to conduct through either tunneling or hopping in localized states near Fermi level in low temperature regime, and they need $0.28 \mathrm{eV}$ to conduct through jumping in band tail states or through the extended states. The values of $\Delta \mathrm{E}_{1}$ and $\Delta \mathrm{E}_{2}$ (DC) for a-C:H:N film grown at NPP of $76.1 \%$ (sample S-4) are found to be $0.19 \mathrm{eV}$ and $0.32 \mathrm{eV}$, respectively. Here, $\Delta \mathrm{E}_{1}$ is the highest among nitrogen incorporated a-C:H:N films (among samples S-2, S-3, and $\mathrm{S}-4)$. We believe that conduction at higher temperature is due to hopping in band tail states.

We have also estimated the $\Delta \mathrm{E}_{1}$ and $\Delta \mathrm{E}_{2}$ from $\mathrm{PC}$ versus inverse of temperature plots. The $\Delta \mathrm{E}_{1}$ and $\Delta \mathrm{E}_{2}$ (PC) were found to be $0.26 \mathrm{eV}$ and $0.36 \mathrm{eV}$ for sample $\mathrm{S}-1$, $0.147 \mathrm{eV}$ and $0.362 \mathrm{eV}$ for sample $\mathrm{S}-2,0.142 \mathrm{eV}$ and $0.281 \mathrm{eV}$ for sample S-3, and $0.123 \mathrm{eV}$ and $0.308 \mathrm{eV}$ for sample $\mathrm{S}-4$. Here, the cause of modification of activation energy for different samples was not only the shifting of Fermi level and a change to the electronic states with the introduction of nitrogen but also the change of states under exposure of light of intensity $100 \mathrm{~mW} / \mathrm{cm}^{2}$. The DC and PC of a-C:H:N films were also correlated with Tauc band gap, which is shown in Fig. 9. The DC and PC both varied inversely with band gap, i.e., lower the band gap greater the DC and PC. This may be due to the fact that low band gap was obtained at higher NPPs, and higher NPPs not only shifted the Fermi level towards the conduction band but also enhanced the conducting $\mathrm{sp}^{2}$ bonding.

\section{G. Analysis of the electrical characteristics of a-C:H:N/p-Si devices}

The current-voltage (I-V) characteristics of different a$\mathrm{C}: \mathrm{H}: \mathrm{N} / \mathrm{p}-\mathrm{Si}$ heterojunction diodes in a sandwich structure configuration, where $\mathrm{Al}$ dots of diameter $1 \mathrm{~mm}$ are used as a metal contacts on a-C:H:N as well as on p-Si, were recorded and shown in Fig. 10(a). These I-V curves clearly revealed the nonlinear behaviour, and hence the creation of rectifying circuits in the heterojunction has been realized. Furthermore, with the increasing nitrogen content not only does the current increases but also the diode turn-on voltage $\left(\mathrm{V}_{\mathrm{on}}\right)$ decreases. In sample S-1 (NPP 0\%), $\mathrm{V}_{\text {on }}$ was found to be $\sim 3 \mathrm{~V}$, which shifted to $\sim 2.5 \mathrm{~V}$ in sample S-2 (NPP 44.4\%). In samples S-3 (NPP 66.6\%) and S-4 (NPP 76.1\%) again $\mathrm{V}_{\text {on }}$ shifted towards lower values and attained the magnitude as $\sim 2 \mathrm{~V}$ and $\sim 1.5 \mathrm{~V}$, respectively. The plots of $\log$ I versus $\mathrm{V}$ for different a-C:H:N films revealed two different modes of conduction in which at higher voltage regime the curves were bent. Hence, I-V curves were fitted for log current versus voltage ${ }^{1 / 2}$ (log I versus $\mathrm{V}^{1 / 2}$ ), which as shown in Fig. 10(b), to analyze electron

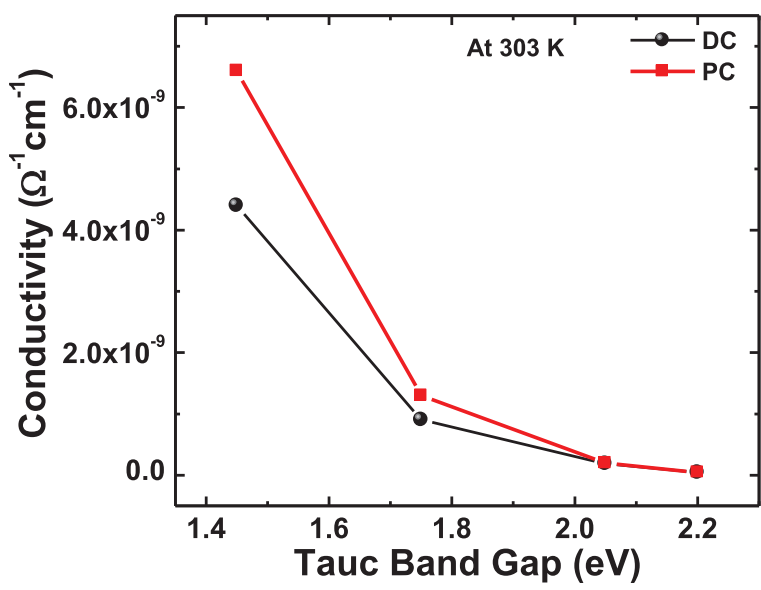

FIG. 9. Variation of conductivity (DC and PC) at $303 \mathrm{~K}$ with Tauc band gap. 

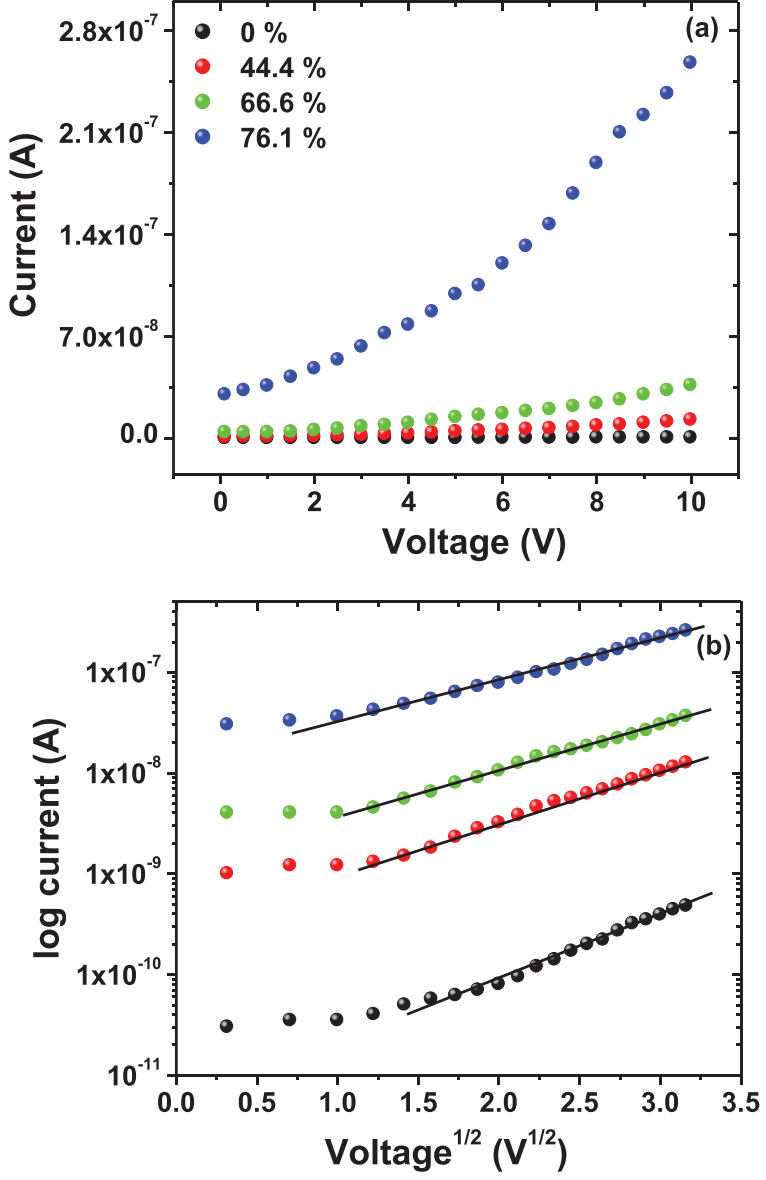

FIG. 10. (a) I-V characteristics and (b) Poole-Frenkel model (log I versus $\mathrm{V}^{1 / 2}$ ) of different a-C:H:N films.

transport. The investigation suggests that transport is bulk limited, not ruled by differences at the barrier heights at the contact. Therefore, occurrence of Poole-Frenkel model at higher voltage was realized, i.e., the conduction at higher voltage (or field) regime took place due to carriers trapped in the localized states. Recently, we have also observed Poole-Frenkel mechanism in a low pressure grown a-C:H:N/p-Si and a-C:H/ $\mathrm{n}$-Si heterojunction diodes. ${ }^{5}$ Khan et al. ${ }^{25}$ have found the Poole-Frenkel behaviour in low mass implanted amorphous carbon films and Miyajima et $a l^{26}$ have reported the PooleFrenkel mechanism in pulse laser deposited nitrogen incorporated amorphous carbon films.

\section{CONCLUSIONS}

The a-C:H:N films were deposited under various NPPs from $0 \%$ to $76.1 \%$ using RF-PECVD technique and were studied for their electrical, optical, mechanical, and structural properties. The introduction of nitrogen in these films enhanced the graphite-like $\mathrm{sp}^{2}$ bonding that was confirmed by XPS, FTIR, and micro Raman analyses. The increase in nitrogen content or NPP reduced the residual stress, tuned the Tauc band gap towards lower values, and improved the conductivity of these films with a reduction in hardness. Photoconductivity has also been observed in these films. An enhanced conductivity with increasing nitrogen content was discussed within a band model that revealed the shifting of Fermi level towards conduction band. The I-V characteristics clearly reveal the rectifying behaviour and their current continuously enhanced with increasing nitrogen content. Analysis of the electrical characteristics also infers that electrical transport at higher voltages (or fields) in these films follow the Poole-Frenkel transport mechanism. Finally, observed excellent properties in high pressure deposited a-C:H:N films are very encouraging and may enhance its industrials applications as the present process is simple, cost effective and produces high throughput.

\section{ACKNOWLEDGMENTS}

The authors are grateful to the Director, National Physical Laboratory, New Delhi (India) for his kind support and encouragement. We acknowledge Ishpal, Dr. O. S. Panwar, and C. M. S. Rauthan for their kind support. Author N.D. acknowledges CSIR, Government of India for providing financial support through SRF fellowship.

${ }^{1}$ A. Y. Liu and M. L. Cohen, Science 245, 841 (1989).

${ }^{2}$ S. R. P. Silva, J. Robertson, G. A. J. Amaratunga, B. Rafferty, L. M. Brown, J. Schwan, D. F. Franceschini, and G. Mariotto, J. Appl. Phys. 81, 2626 (1997).

${ }^{3}$ J. Schwan, V. Batori, S. Ulrich, and H. Ehrhardt, J. Appl. Phys. 84, 2071 (1998).

${ }^{4}$ C. Godet, N. M. J. Conway, J. E. Bouree, K. Bouamra, A. Grosman, and C. Ortega, J. Appl. Phys. 91, 4154 (2002).

${ }^{5}$ N. Dwivedi, S. Kumar, and H. K. Malik, J. Appl. Phys. 11, 014908 (2012).

${ }^{6}$ N. Dwivedi, S. Kumar, and H. K. Malik, ACS Appl. Mater. Interfaces 3, 4268 (2011).

${ }^{7}$ V. S. Veerasamy, G. A. J. Amaratunga, J. S. Park, W. I. Milne, H. S. Mckenzie, and D. S. Mckenzie, Appl. Phys. Lett. 64, 2297 (1994).

${ }^{8}$ K. M. Krishna, M. Umeno, Y. Nukaya, T. Soga, and T. Jimbo, Appl. Phys. Lett. 77, 1472 (2000).

${ }^{9}$ N. Konofaos, E. Evangelou, and C. B. Thomas, J. Appl. Phys. 84, 4634 (1998).

${ }^{10}$ X. Gao, X. Zhang, C. Wan, X. Zhang, L. Wu, and X. Tan, Appl. Phys. Lett. 97, 212101 (2010)

${ }^{11}$ I. Lazar and G. Lazar, J. Non-Cryst. Solids 352, 2096 (2006).

${ }^{12}$ S. R. P. Silva and J. D. Carey, Diamond Relat. Mater. 12, 151 (2003).

${ }^{13}$ C. Ronning, U. Greismeier, M. Groos, H. C. Hofsass, R. G. Downing, and G. P. Lamaze, Diamond Relat. Mater. 4, 666 (1995).

${ }^{14}$ C. Godet and J. P. Kleider, J. Mater. Sci.: Mater. Electron. 17, 413 (2006).

${ }^{15}$ S. J. Henley, J. D. Carey, and S. R. P. Silva, Appl. Phys. Lett. 85, 6236 (2004).

${ }^{16}$ S. Kumar, N. Dwivedi, C. M. S. Rauthan, and O. S. Panwar, Vacuum 84, $882(2010)$.

${ }^{17}$ C. D. Wagner, W. M. Riggs, L. E. Davis, J. F. Moulder, and G. E. Muilenberg, Handbook of X-ray Photoelectron Spectroscopy (Perkin Elmer Corporation, 1979).

${ }^{18}$ A. C. Ferrari and J. Robertson, Philos. Trans. R. Soc. London 362, 2477 (2004).

${ }^{19}$ F. L. Freire, Jr., J. Non-Cryst. Solids 304, 251 (2002).

${ }^{20}$ J. Robertson, Mater. Sci. Eng. R. 37, 129 (2002).

${ }^{21}$ M. Umeno and S. Adhikary, Diamond Relat. Mater. 14, 1973 (2005).

${ }^{22}$ S. Liu, G. Wang, and Z. Wang, J. Non-Cryst. Solids 353, 2796 (2007).

${ }^{23}$ P. R. Somani, A. Yoshida, R. A. Afre, S. Adhikari, T. Soga, and M. Umeno, Phys. Status Solidi A 203, 1982 (2006).

${ }^{24}$ J. D. Carey and S. R. P. Silva, Phys. Rev. B 70, 235417 (2004).

${ }^{25}$ R. U. A. Khan, J. D. Carey, and S. R. P. Silva, Phys. Rev. B 63, 121201(R) (2001).

${ }^{26}$ Y. Miyajima, S. J. Henley, G. Adamopoulos, V. Stolojan, E. G. Caurel, B. Drevillon, J. M. Shannon, and S. R. P. Silva, J. Appl. Phys. 105, 073521 (2009). 